\title{
Multipoint observations of compressional Pc5 pulsations in the dayside magnetosphere and corresponding particle signatures
}

\author{
Galina Korotova $^{1,2}$, David Sibeck ${ }^{3}$, Mark Engebretson ${ }^{4}$, Michael Balikhin ${ }^{5}$, Scott Thaller $^{6}$, Craig Kletzing ${ }^{7}$, \\ Harlan Spence $^{8}$, and Robert Redmon ${ }^{9}$ \\ ${ }^{1}$ Institute for Physical Science and Technology (IPST), University of Maryland, College Park, MD, USA \\ ${ }^{2}$ Pushkov Institute of Terrestrial Magnetism, Ionosphere and Radio Wave Propagation (IZMIRAN), \\ Russian Academy of Sciences, Troitsk, Moscow, Russia \\ ${ }^{3}$ NASA Goddard Space Flight Center (GSFC), Code 674, Greenbelt, MD, USA \\ ${ }^{4}$ Department of Physics, Augsburg University, Minneapolis, MN, USA \\ ${ }^{5}$ Department of Automatic Control and Systems Engineering, University of Sheffield, Sheffield, UK \\ ${ }^{6}$ Laboratory for Atmospheric and Space Physics (LASP), University of Colorado, Boulder, CO, USA \\ ${ }^{7}$ Department of Physics and Astronomy, Iowa University, Iowa City, IA, USA \\ ${ }^{8}$ Institute for the Study of Earth, Oceans, and Space (EOS), University of New Hampshire, Durham, NH, USA \\ ${ }^{9}$ Solar and Terrestrial Physics division, National Geophysical Data Center (NGDC/NOAA), Boulder, CO, USA
}

Correspondence: Galina Korotova (gkorotov@umd.edu)

Received: 1 May 2020 - Discussion started: 26 June 2020

Revised: 25 October 2020 - Accepted: 5 November 2020 - Published: 21 December 2020

\begin{abstract}
We use Van Allen Probes (Radiation Belt Storm Probes A and B, henceforth RBSP-A and RBSP-B) and GOES-13 and GOES-15 (henceforth G-13 and G-15) multipoint magnetic field, electric field, plasma, and energetic particle observations to study the spatial, temporal, and spectral characteristics of compressional Pc5 pulsations observed during the recovery phase of a strong geomagnetic storm on 1 January 2016. From $\sim$ 19:00 to 23:02 UT, successive magnetospheric compressions enhanced the peak-to-peak amplitudes of Pc5 waves with $4.5-6.0 \mathrm{mHz}$ frequencies from 0 2 to $10-15 \mathrm{nT}$ at both RBSP-A and RBSP-B, particularly in the prenoon magnetosphere. Poloidal Pc4 pulsations with frequencies of $\sim 22-29 \mathrm{mHz}$ were present in the radial $\mathrm{Bx}$ component. The frequencies of these Pc4 pulsations diminished with increasing radial distance, as expected for resonant Alfvén waves standing along field lines. The GOES spacecraft observed Pc5 pulsations with similar frequencies to those seen by the RBSP but Pc4 pulsations with lower frequencies.

Both RBSP-A and RBSP-B observed frequency doubling in the compressional component of the magnetic field during the Pc5 waves, indicating a meridional sloshing of the equatorial node over a combined range in $Z_{\mathrm{SM}}$ from 0.25
\end{abstract}

to $-0.08 \mathrm{Re}$, suggesting that the amplitude of this meridional oscillation was $\sim 0.16 \mathrm{Re}$ about an equatorial node whose mean position was near $Z_{\mathrm{SM}}=\sim 0.08 \mathrm{Re}$. RBSP-A and RBSP-B HOPE (Helium Oxygen Proton Electron) and MagEIS (Magnetic Electron Ion Spectrometer) observations provide the first evidence for a corresponding frequency doubling in the plasma density and the flux of energetic electrons, respectively. Energetic electron fluxes oscillated out of phase with the magnetic field strength with no phase shift at any energy. In the absence of any significant solar wind trigger or phase shift with energy, we interpret the compressional Pc5 pulsations in terms of the mirror-mode instability.

\section{Introduction}

Ultralow frequency (ULF) pulsations - with periods of $100 \mathrm{~s}$ or greater, with high azimuthal wave numbers $(m)$, with magnetic field perturbations in the radial direction, and with electric field perturbations in the azimuthal direction - within the Earth's magnetosphere are typically poloidal waves (Sugiura and Wilson, 1964). According to Elkington et al. (2003), energetic particles with drift frequencies of 6.7-22 and 1.7- 
$6.7 \mathrm{mHz}$ can readily interact with corresponding high- $m$ poloidal Pc4 and Pc5 pulsations. Because the atmosphere and ionosphere screen these high- $m$ waves from the ground, they can only be studied with the help of satellite observations. Past studies of Pc4 and Pc5 pulsations with significant compressional components employed observations from locations at or near geosynchronous orbit (e.g., Dai et al., 2013). Higbie et al. (1982) and Nagano and Araki (1983) showed that long-lasting compressional Pc5 pulsations occur most frequently in the dayside magnetosphere during the recovery phase of magnetic storms. Storm-time Pc5 pulsations occur in the afternoon sector between 12:00 and 18:00 LT (local time) following injections of ring current particles (Kokubun, 1985).

A number of studies have examined compressional Pc5 waves outside geostationary orbit. According to these studies, compressional Pc5 waves were observed in the dawn (Hedgecock, 1976), dusk (Constantinescu et al., 2009), and noon (Takahashi et al., 1985) sectors. Zhu and Kivelson (1991) reported that intense compressional waves are a persistent feature on both flanks of the magnetosphere. Compressional Pc5 pulsations occur within $\sim 20^{\circ}$ latitude of the magnetic equator (Vaivads et al., 2001). They have wavelengths of several radii (Walker et al., 1982) and often exhibit harmonics. Elkington et al. (2003) noted that poloidal and compressional modes are far more effective for the radial transport of energetic particles than the toroidal mode. Two methods are used to identify the harmonic mode of a poloidal oscillation. The first compares the phase difference between the radial component of the magnetic field and the azimuthal component of the electric field (Takahashi et al., 2011). The second compares observed wave frequencies with the eigenfrequencies predicted by theory (Cummings, 1969). The multisatellite study of Takahashi et al. (1987a) showed that a compressional Pc5 wave had an antisymmetric standing structure.

Compressional Pc5 pulsations have been ascribed to numerous excitation mechanisms. They can be produced by internal and external processes. It is supposed that the solar wind is the main external source for pulsations produced by the Kelvin-Helmholtz (KH) instability at the magnetopause or the inner edge of the low-latitude boundary layer (e.g., Guo et al., 2010). Observations indicating enhanced rates of Pc5 occurrence during periods of greater solar wind velocity support this model (e.g., Engebretson et al., 1998). Transient variations in the dynamic pressure of the solar wind or foreshock (e.g., Wang et al., 2018; Shen et al., 2018) that cause abrupt changes in the magnetic field strength in the magnetosphere and sudden impulses in the ionosphere (e.g., Zhang et al., 2010; Sarris et al., 2010) provide another possible trigger for Pc5 pulsations. External pressure impulses can cause compressional oscillations of the magnetosphere with discrete eigenfrequencies, known as global modes or cavity/waveguide modes (Samson et al., 1992). Periodic solar wind dynamic pressure variations directly drive some com- pressional magnetospheric magnetic field oscillations (e.g., Kepko and Spence, 2003; Motoba et al., 2003). Takahashi and Ukhorskiy (2008) considered solar wind pressure variations as the main external driver of Pc5 pulsations observed at geosynchronous orbit in the dayside magnetosphere.

Internal generation mechanisms for compressional Pc5 pulsations include the drift-bounce resonant instability which occurs for particles with resonance drift and bounce periods (Southwood et al., 1969) and the drift-mirror instability in the presence of strong temperature anisotropies (Chen and Hasegawa, 1991). In high $\beta$ plasmas ( $\beta$ is the plasma pressure divided by the magnetic pressure), these mechanisms favor antisymmetric waves (Cheng and Lin, 1987).

One possible generation mechanism for compressional Pc5 pulsations at geosynchronous orbit is a drift-mirror instability of ring current particles (e.g., Lanzerotti et al., 1969). While the observed anticorrelated magnetic field strength and ion flux oscillations are expected for a drift-mirror wave (Kremser et al., 1981), the instability criterion is generally not satisfied (Pokhotelov et al., 1986). One possible reason for the lack of consistency between theory and observation might be because the real geometry of the magnetosphere is not taken into account (Cheng and Lin, 1987). Compressional pulsations are often accompanied by pulsations in particle fluxes (Kremser et al., 1981; Liu at al., 2016). Particle observations can provide useful information on the spatial and wave structure of ULF pulsations. Lin et al. (1976) explained flux oscillations as the adiabatic motion of particles in a magnetohydrodynamic wave. Kivelson and Southwood (1985) studied charged particle behavior in compressional ULF waves and showed that "a mirror effect" is the dominant cause for particle flux modulations. Finite gyroradius effects enable detection of gradients in particle flux associated with waves (e.g., Korotova et al., 2013).

We use multipoint magnetic field, plasma, and energetic particle observations from RBSP-A and RBSP-B (Radiation Belt Storm Probes) and G-13 and G-15 (GOES) to study the spatial, temporal, and spectral characteristics of compressional Pc5 pulsations observed deep within the magnetosphere during the recovery phase of the strong magnetic storm which began on 31 December 2015. We investigate the type of pulsation (compressional versus transverse), their harmonic mode, and their latitudinal nodal structure. We focus on the properties of double-frequency pulsations that occurred in the vicinity of the geomagnetic equator. We demonstrate that the energetic particles respond directly to the compressional Pc5 pulsations and also exhibit a double-frequency oscillation. We search for possible solar wind triggers and test two possible generation mechanisms: drift-bounce resonance and mirror instability. The paper is organized as follows. Section 2 describes the instruments and resources. Section 3 presents the solar wind and interplanetary magnetic field (IMF) conditions. Section 4 provides an analysis of these waves and their generation mechanisms. 


\section{Resources}

The Van Allen Probes mission can be used to study the geospace response to a fluctuating solar wind. The mission began in August 2012 with a twin spacecraft launch into similar $10^{\circ}$ inclination orbits with perigee altitudes slightly greater than $600 \mathrm{~km}$ and apogee altitudes just beyond $30000 \mathrm{~km}$ (Mauk et al., 2012). The spacecraft carry instruments that measure electromagnetic fields, ultralow frequency waves, and charged particle populations deep within the magnetosphere. This paper employs observations of the most abundant ion components as well as electrons, over the $0.001-50 \mathrm{keV}$ energy range, of the core plasma populations from the HOPE instrument; populations of $20-4000 \mathrm{keV}$ ions and electrons from the MagEIS instrument (Blake et al., 2013) in the Energetic Particle, Composition, and Thermal Plasma (ECT) suite (Spence et al., 2013); and fluxes of ions over the energy range from $\sim 20$ to $\sim 1 \mathrm{MeV}$ and electrons over the energy range from $\sim 25$ to $\sim 1 \mathrm{MeV}$ (RBSPICE, Radiation Belt Storm Probes Ion Composition Experiment) (Mitchell et al., 2013) in conjunction with observations from the magnetometer in the Electric and Magnetic Field Instrument Suite and Integrated Science (EMFISIS) suite (Kletzing et al., 2013) and the Electric Field and Waves (EFW) instrument (Wygant et al., 2013). We examine electric and magnetic field measurements with 11 and $4 \mathrm{~s}$ time resolutions, respectively, and differential particle flux observations with $\sim 11 \mathrm{~s}$ (spin period) time resolution. The data are provided by NASA GSFC's CDAWeb (Coordinated Data Analysis Web) in the MGSE (modified GSE, with GSE meaning geocentric solar ecliptic) coordinate system. We use magnetic field data from G-13 and G-15 with $0.5 \mathrm{~s}$ time resolution (Singer et al., 1996). Finally, we employ Wind (spacecraft) solar wind magnetic field and 3DP plasma data with $3 \mathrm{~s}$ time resolution (Lepping et al., 1995; Lin et al., 1995).

\section{Orbits, solar wind, and geomagnetic conditions}

Figure 1 presents the $\mathrm{Bz}$ component of the interplanetary magnetic field observed at Wind, and geomagnetic activity indices (Dst and AE) obtained from the OMNI database (upper panels; (http://omniweb.gsfc.nasa.gov, last access: 30 April 2020), from 12:00 UT on 30 December to 00:00 UT on 2 January 2016. The bottom panels show Wind observations of the magnetic field components, total magnetic field strength, cone angle, pressure, plasma density, and velocity from 16:00 UT on 1 January 2016 to 00:00 UT on 2 January 2016 during which time the spacecraft moved from GSM $(X, Y, Z)=(194.7,20.1,-12.5) \operatorname{Re}$ to $(194.8$, 23.6, -7.4) Re (where GSM represents the geocentric solar magnetospheric system and Re represents Earth radius). The pulsation events to be studied here occurred late on 1 January 2016, following a prolonged period of strongly southward IMF orientation and geomagnetic activity. A sub-

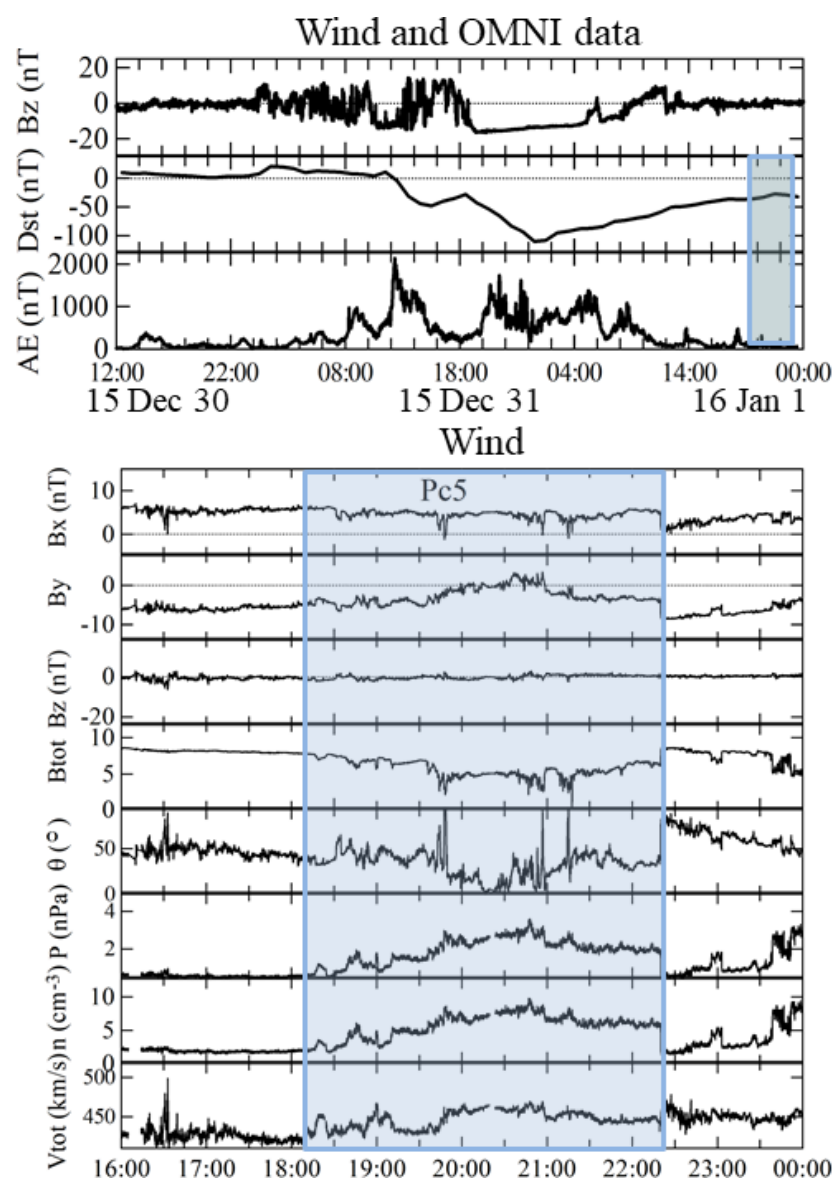

Figure 1. Bz component of the magnetic field observed at Wind and geomagnetic activity indices (Dst and $\mathrm{AE}$ ) obtained from the OMNI database (upper panels) from 12:00 UT on 30 December to 00:00 UT 2 January 2016. The bottom panels show Wind observations of the magnetic field components, total magnetic field strength, cone angle, pressure, plasma density, and velocity from 16:00 UT on 1 January 2016 to 00:00 UT on 2 January 2016. Shading highlights intervals when magnetospheric spacecraft observed Pc5 compressional pulsations.

stantial increase in the solar wind dynamic pressure early on 31 December was followed by a strong southward IMF that persisted from 19:00 UT on 31 December 2015 until 09:00 UT on 1 January 2016. A strong electrojet with AE index greater than $2100 \mathrm{nT}$ at 12:36 UT on 31 December 2015 was followed by two moderate substorms that enhanced $\mathrm{AE}$ at $\sim 14: 00$ and 18:45 UT on 1 January 2016. The Dst index responded by reaching a value as low as $-110 \mathrm{nT}$ at 00:30 UT on 1 January 2016. Shading highlights the interval from $\sim$ 19:00 to 23:02 UT late in the recovery phase and late in the day on 1 January 2016 when the Van Allen Probes and GOES spacecraft observed the strong compressional Pc5 pulsations of interest to this study.

The latter interval (bottom panels) was marked by strong variations in the solar wind dynamic pressure. Shading marks 

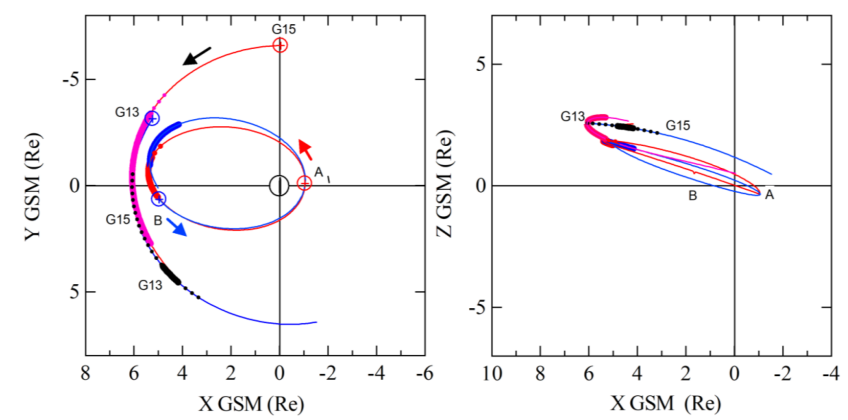

Figure 2. Trajectories of RBSP-A (red), RBSP-B (blue), G-13 (black), and G-15 (purple) from 15:00 to 24:00 UT on 1 January 2016 in the X-Y and X-Z GSM planes. Open circles mark the beginning of the spacecraft trajectories which are duskward for the GOES spacecraft and duskward at apogee for the Van Allen Probes. The thick line segments indicate the locations of the spacecraft at the times when compressional Pc5 magnetic field pulsations occurred. Dots mark their locations where weak pulsations $(\mathrm{A}<5 \mathrm{nT})$ occurred.

an interval of depressed magnetic field strengths and generally anticorrelated enhanced densities, velocities, and solar wind dynamic pressures. The cone angle, $\theta$, defined as the angle between the IMF and the Sun-Earth line was less than $45^{\circ}$ during this interval. The magnetic field was briefly aligned with the Sun-Earth line $(\mathrm{Bx})$ at the center of the interval from 20:00-21:00 UT. For most of the $\sim 4 \mathrm{~h}$ long shaded interval, IMF Bx (By) was predominantly positive (negative) and the $\mathrm{Bz}$ component remained almost constant near $0 \mathrm{nT}$, indicating a spiral and equatorial IMF configuration. The total magnetic field strength decreased from $7.9 \mathrm{nT}$ at 18:00 UT to $2.2 \mathrm{nT}$ at 19:48 UT, and the solar wind velocity and dynamic pressure increased from $426 \mathrm{~km} / \mathrm{s}$ and $0.62 \mathrm{nPa}$ at 18:00 UT to $457 \mathrm{~km} / \mathrm{s}$ and to $3.37 \mathrm{nPa}$ at 20:47 UT, respectively. At $\sim 22: 20 \mathrm{UT}$ almost all parameters returned to their initial undisturbed values.

Figure 2 presents RBSP-A, RBSP-B, G-13 (MLT is $\sim \mathrm{UT}-5$, where MLT represents magnetic local time), and G-15 (MLT is UT-9) trajectories from 15:00 to 24:00 UT on 1 January 2016 in the X-Y and X-Z GSM planes. Open circles mark the beginning of the spacecraft trajectories which are duskward for the GOES spacecraft and duskward at apogee for the Van Allen Probes. All of the spacecraft were north of the equator when in the dayside magnetosphere. The thick line segments (dots) indicate the locations of the spacecraft at the times when (weak) Pc5 magnetic field pulsations occurred.

Figure 3 compares lagged Wind solar wind dynamic pressure variations with G-13 and G-15 observations of the dayside magnetospheric magnetic field. The arrows connect enhancements of the solar wind dynamic pressure to corresponding compressions of the magnetosphere. To determine the lag time between the Wind and GOES-15 observations, we related individual magnetosphere compressions to corre- sponding dynamic pressure variations. Additionally, we confirmed these empirically derived lag times with simple ballistic estimates based on the solar wind velocity and the distance of Wind from Earth. It is relatively easy to associate the GOES magnetic field enhancements with corresponding features in the solar wind dynamic pressure at the beginning and the end of the interval but less easy from 19:50 to $21: 20$ UT corresponding to $\sim 20: 45$ and $22: 15$ UT at the GOES spacecraft. The lag time from Wind to the Earth is not uniform and depends on IMF orientation. At the beginning and end of the interval, when the IMF was spiral $(\mathrm{Bx}>0$, $\mathrm{By}<0$ ), the lag was in the range of $\sim 46$ to $58 \mathrm{~min}$. Consistent with expectations, the lag became greater for the interval from $\sim$ 19:50 to 21:20 UT when the IMF was nearly radial (By and $\mathrm{Bz} \sim 0 \mathrm{nT}$ ). The reasonable correspondence of the magnetosphere compressions to solar wind dynamic pressure variations demonstrates that Wind was a good monitor for solar wind conditions and that a series of pressure enhancements were applied to the magnetosphere during the interval of interest. Pc5 pulsation amplitudes at G-13 and G-15 were greater during the interval of enhanced solar wind dynamic pressure and magnetospheric magnetic field strengths than they were at earlier and later times.

\section{Pulsation observations}

\subsection{Spatial characteristics of Pc5 pulsations}

Consider the spatial, temporal, and spectral characteristics of the compressional Pc5 pulsations. Figure 4a shows G-13 and G-15 observations of the total magnetic field strength from 18:00 to 24:00 UT. The spacecraft observed long-duration Pc5 pulsations over a wide longitudinal region in the pre- and postnoon magnetosphere from 10:00 to 15:20 MLT (Fig. 2). G-15 observed weak, less than $\sim 5 \mathrm{nT}$ amplitude, Pc5 waves from 18:28 to 19:04 UT prior to the main event. During the main event from 19:04 to 23:00 UT, the magnetosphere was compressed (Fig. 3), magnetic field strengths increased, and the amplitude of these waves increased to values ranging from 10 to $16 \mathrm{nT}$ with peak amplitudes prior to local noon. G-13 observed weak Pc5 pulsations with amplitudes of 2$4 \mathrm{nT}$ throughout most of the time interval from 16:40 UT (not shown) to 21:00 UT. During the interval from 19:34 UT ( $\sim 14: 45$ MLT) to 20:10 UT ( $\sim 15: 20$ MLT), the pulsations reached slightly stronger amplitudes of 5-8 nT. At 23:02 UT all Pc5 wave activity at both GOES stopped.

Figure $4 \mathrm{~b}$ shows the RBSP-A and RBSP-B total magnetic field strength from 18:40 to 21:10 UT and from 20:40 to 23:10 UT, respectively, on 1 January 2016. Taken together, RBSP-A and RBSP-B observed Pc5 pulsations that occupied the inner dayside magnetosphere from 5.26 to $5.75 \mathrm{Re}$ and from 09:56 to 12:44 MLT (Fig. 2). Prior to the arrival of the strong solar wind dynamic pressure variations from 18:15 to 18:55 UT, RBSP-A observed very weak pulsations 


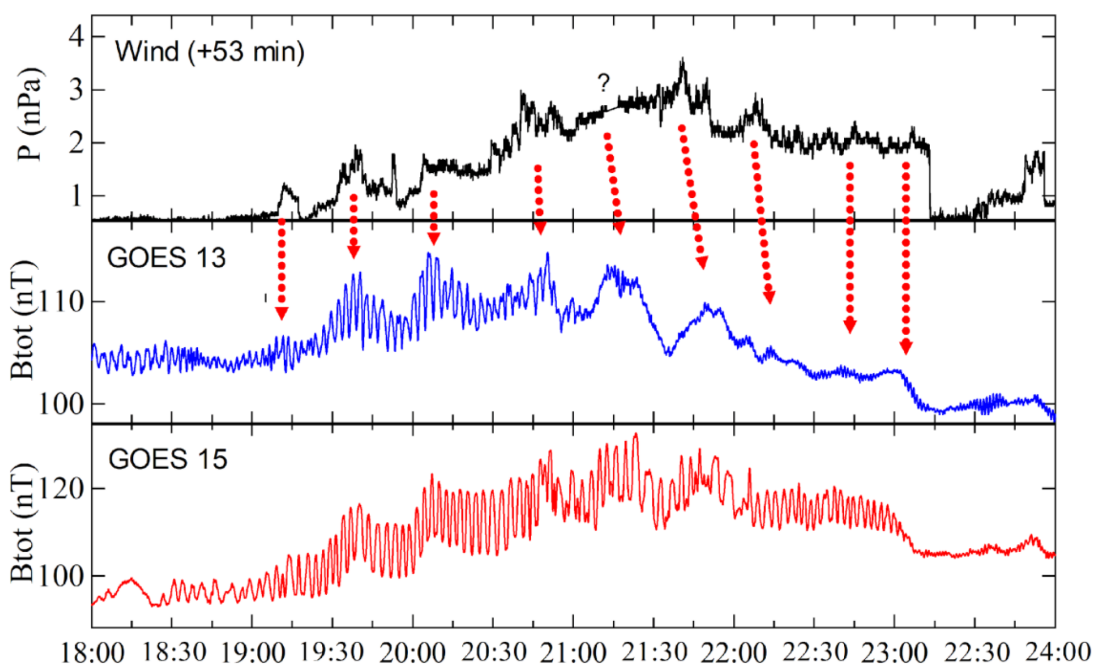

Figure 3. Observations of the solar wind dynamic pressure at Wind (time shifted) and the total magnetic field strength at G-13 and G-15 from 18:00 to 24:00 UT. The arrows connect enhancements of the solar wind dynamic pressure to corresponding compressions of the magnetosphere.

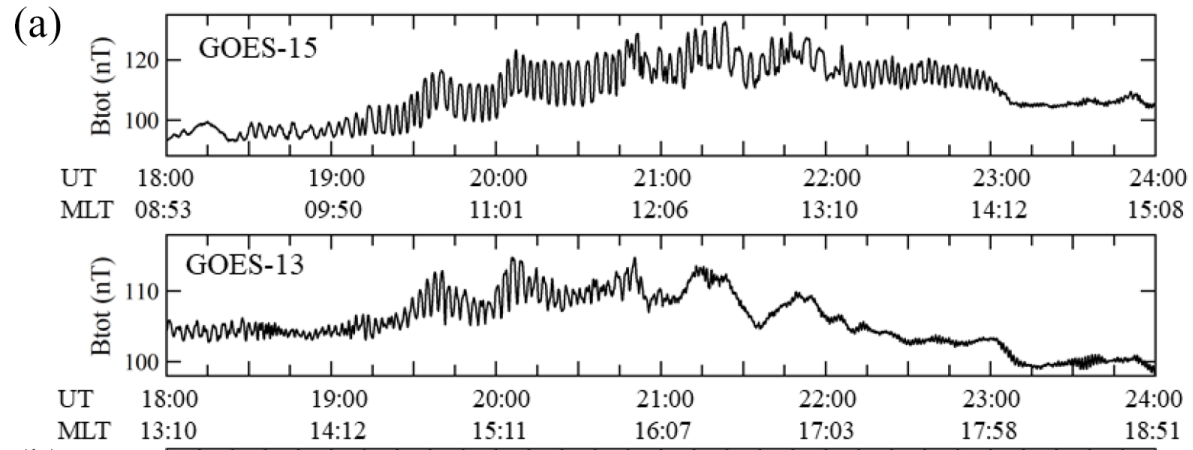

(b)
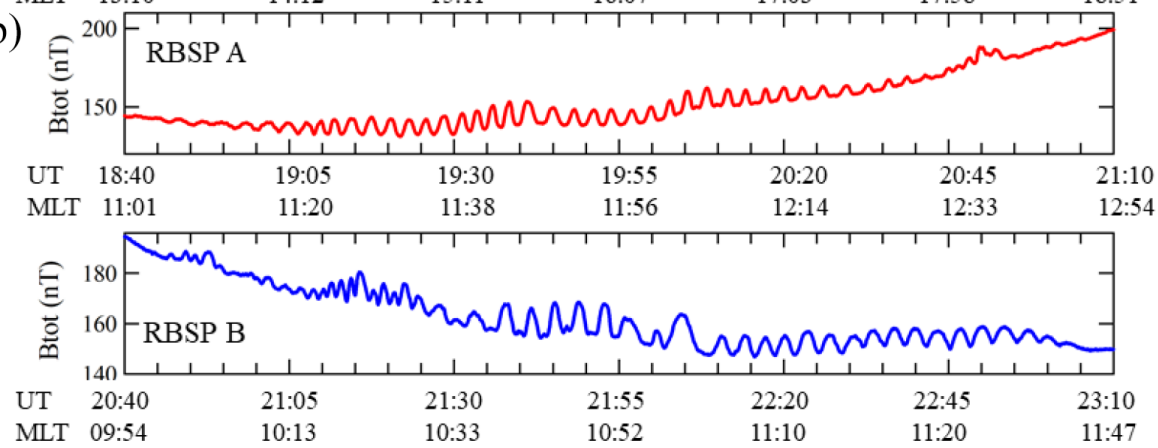

Figure 4. (a, b) G-15 and G-13 (a) total magnetic field strength from 18:00 to 24:00 UT on 1 January 2016. RBSP-A and RBSP-B (b) total magnetic field strength from 18:40 to 21:10 UT and from 20:40 to 23:10 UT on 1 January 2016, respectively, Beneath the panels are listed the universal time (UT) and magnetic local time (MLT).

with Pc5 periods and amplitudes of 1-3nT (not visible at this scale). After the compression of the magnetosphere just after 19:00 UT, the pulsation amplitude at RBSP-A increased to values ranging from 10 to $15 \mathrm{nT}$ with the peak amplitude occurring prior to local noon (Fig. 4b). RBSP-B observed similar compressional Pc5 pulsations from 20:46 UT that ceased simultaneously with the end of the magnetospheric compression at about 23:02 UT.

To determine the type of the Pc5 waves, we converted the magnetic field observations from GSE into the field-aligned coordinate system (FAC). Here the $Z$ axis lies parallel to the locally averaged magnetic field. The $Y$ axis points approximately azimuthally eastward and is transverse to B and to 

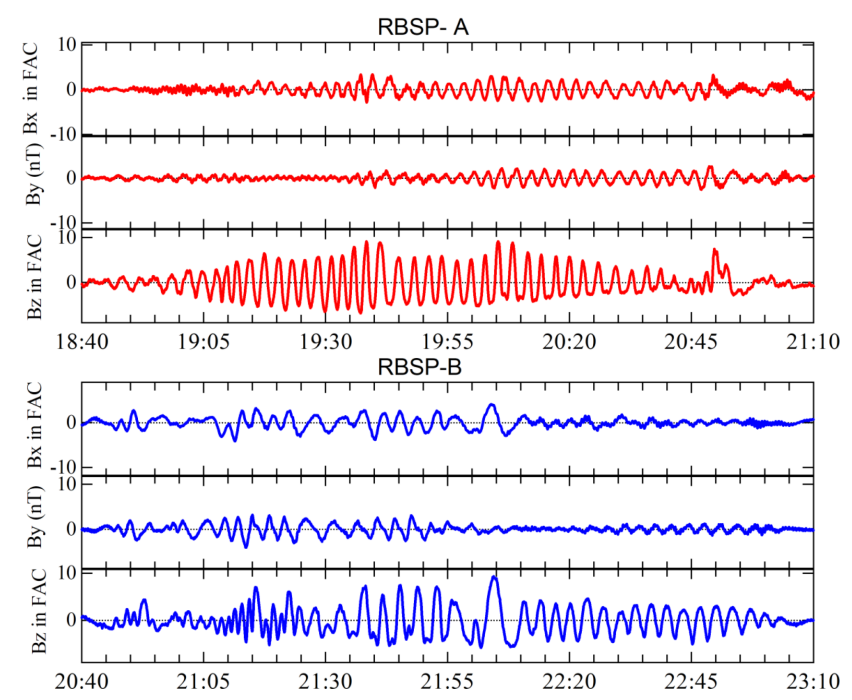

Figure 5. RBSP-A and RBSP-B magnetic field observations in field-aligned coordinates from 18:40 to 21:10 UT and from 20:40 to $23: 10$ UT on 1 January 2016, respectively.

the outward radius vector. The $X$ axis completes the righthanded system and is directed approximately radially outward from Earth. Figure 5 presents RBSP-A and RBSP-B magnetic field observations in FAC. The Bz component is the value of the total magnetic field after subtraction of a $16 \mathrm{~min}$ sliding average. The Pc5 pulsations are observed in all three components, but the amplitudes of the azimuthal By and radial Bx components are rather small and do not exceed $7 \mathrm{nT}$. The compressional $\mathrm{Bz}$ component is much more pronounced for both spacecraft, reaching amplitudes of 14-15 nT before local noon. Consequently, the pulsations are primarily compressional. The $\mathrm{Bz}$ component oscillated out of phase with the Bx component at RBSP-A and in phase at RBSP-B and in quadrature with the By component. Simultaneous RBSP$A$ and RBSP-B electric and magnetic field measurements provide an opportunity to study the mode of the Pc5 waves. Determining the harmonic mode of the Pc5 waves requires us to consider the phase of the azimuthal component of the electric field, Ey, with respect to the radial component of the magnetic field $\mathrm{Bx}$ as a function of latitude (Takahashi et al., 2011). Figure 6 shows that the phase of the Ey component leads that of the Bx component by $90^{\circ}$ at RBSP-A from 19:10 to 20:00 UT; therefore, the Pc5 waves are second harmonic in nature.

\subsection{Spectral characteristics}

We calculated dynamic spectra for the magnetic field pulsations. Figure 7 presents the radial, azimuthal, and compressional components of the dynamic spectra of the magnetic field at RBSP-A and RBSP-B from 18:00 to 21:10 UT and from 20:00 to 23:10 UT on 1 January 2016, respectively. The color bar on the right shows the scale for power for frequen-

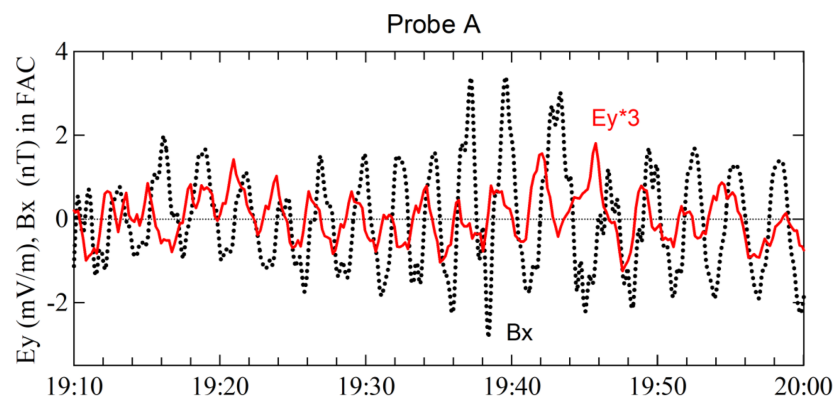

Figure 6. The phase difference between the RBSP-A azimuthal component of the electric field (red curve is boxcar smoothed) and the radial component of the magnetic field $\mathrm{Bx}$ in field-aligned coordinates (dashed curve) from 19:10 to 20:00 UT on 1 January 2016. The amplitude of Ey was multiplied by a factor of 3 to better display the visual effects.

cies ranging from 0 to $41 \mathrm{mHz}$ in each component. The magnetic field exhibited several wideband enhancements at frequencies ranging from 4 to $29 \mathrm{mHz}$. As expected for compressional Pc5 pulsations, both GOES spacecraft observed the strongest power densities in the $\mathrm{Bz}$ component at dominant frequencies of $\sim 4.5-6 \mathrm{mHz}$. Red arrows in the Bz panels of Fig. 7 for RBSP-A and RBSP-B indicate the doublefrequency pulsations at $\sim 5.5$ and $\sim 11 \mathrm{mHz}$. We calculated Fourier spectra for the three components of the RBSP-A and RBSP-B magnetic field in $600 \mathrm{~s}$ sliding-average mean FAC for each $30 \mathrm{~min}$ interval during the event. Figure 8 presents examples of Fourier spectra calculated for the RBSP-A and RBSP-B magnetic field from 19:30 to 20:00 UT and from 22:30 to 23:00 UT, respectively, on 1 January 2016. The red arrows show the dominant frequencies at 5.5 and $5 \mathrm{mHz}$ observed at the two spacecraft, corresponding to periods of 170-200 s. RBSP-A and RBSP-B were situated $3 \mathrm{~h}$ in local time apart; the similar frequencies indicate that conditions in the dayside magnetosphere remained steady for a long time and over a broad region.

In passing, we note the presence of Pc4 pulsations. Returning to Fig. 7, we see enhanced power densities at frequencies of $\sim 22-29 \mathrm{mHz}$ with dominant frequencies from 23 to $27 \mathrm{mHz}$ primarily in the radial Bx component. These can be ascribed to poloidal Pc4 produced simultaneously with the Pc5 but likely with another energy source. The frequencies of the Pc4 pulsations decrease with increasing radial distance, as expected for resonant standing Alfvén waves (Sugiura and Wilson, 1964). Pulsation periods depend upon the magnetic field line length, the magnetic field magnitude, and the ion density. Shorter field line lengths and enhanced magnetic field strengths closer to Earth decrease pulsation periods. Blue arrows in Fig. 8 indicate Pc4 pulsations at $\sim 25-$ $27 \mathrm{mHz}$.

Figure 9 presents dynamic spectra for the G-13 and G-15 magnetic field in FAC from 18:00 to 24:00 UT on 1 January 2016. Spectral power was calculated for frequencies 


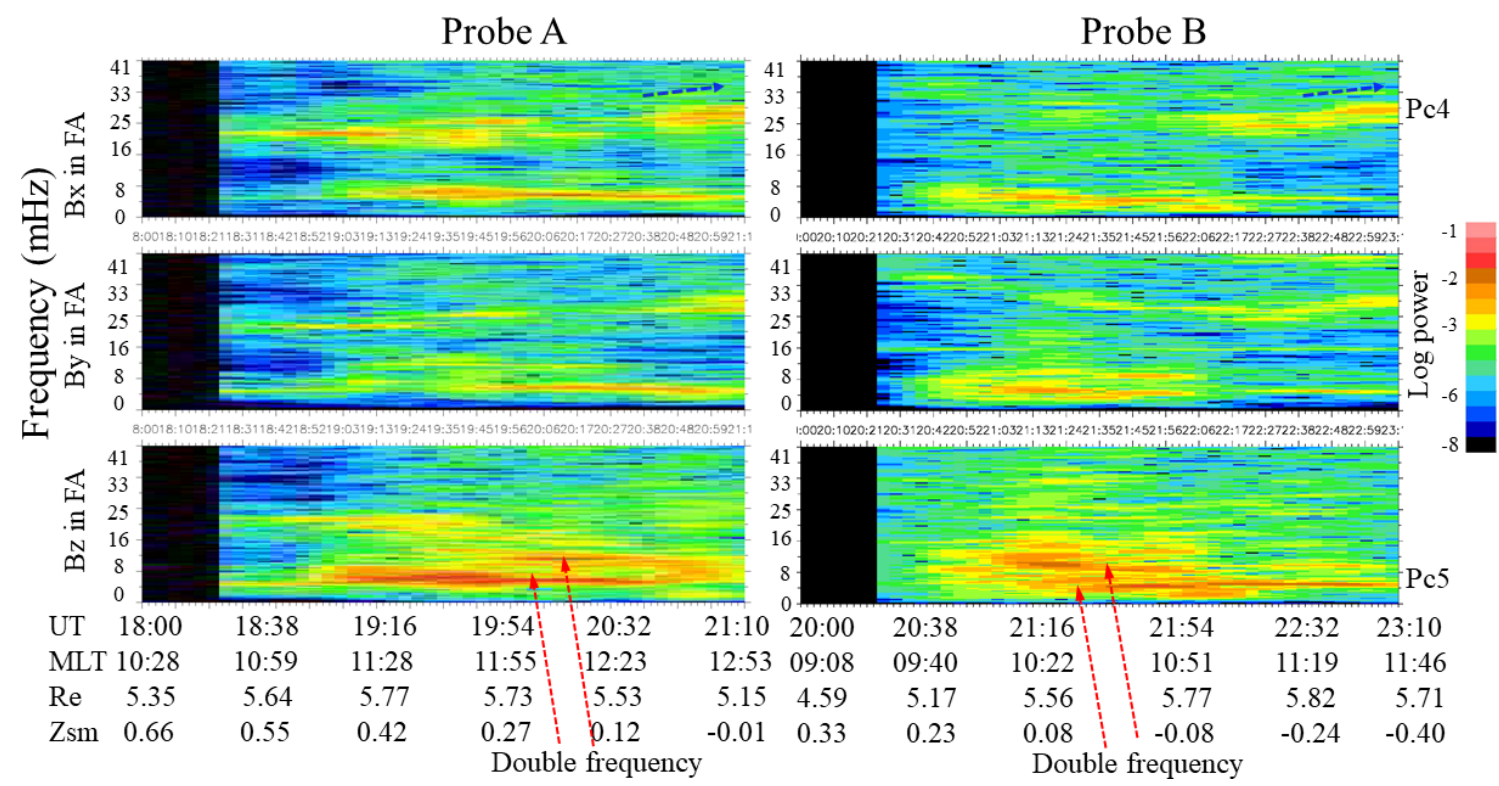

Figure 7. Three-component dynamic spectra of magnetic field data at RBSP-A and RBSP-B from 18:00 to 21:10 UT and from 20:00 to 23:10 UT on 1 January 2016, respectively. Beneath the panels are listed the universal time (UT), magnetic local time (MLT), radius (Re), and $Z$ (SM) in Earth radii.

from 0 to $48 \mathrm{mHz}$. Like the RBSP-A and RBSP-B magnetic field spectra, there are two broad frequency band enhancements corresponding to Pc4 and Pc5 frequencies. The dominant frequencies for the compressional Pc5 pulsations occur from 4.5 to $6.5 \mathrm{mHz}$. These frequencies are similar to those observed by Van Allen Probes, and we suppose that they were generated by the same sources. The Pc4 pulsations are most pronounced in the radial Bx component and display strongest spectral power densities in the frequency range from 13 to $21 \mathrm{mHz}$. These frequencies are lower than those observed by Van Allen Probes, since the GOES spacecraft were located further radially outward from Earth (Sugiura and Wilson, 1964). The frequencies of the long-lasting Pc4 pulsations observed by G-15 depended on local time. They decreased from $20-22 \mathrm{mHz}$ in the prenoon magnetosphere to $14-17 \mathrm{mHz}$ near local noon, perhaps in response to differing conditions (e.g., densities). Takahashi at el. (1984) noted that an increase in plasma mass density from morning to afternoon is typical at geosynchronous orbit. Since the frequencies of the Pc4 pulsations depended on local time and radial distance from Earth, their sources must be more localized than those for the Pc5 pulsations.

\subsection{Particle signatures}

Energetic particle observations provide further information concerning this event. We inspected RBSP-A and RBSP-B MagEIS observations of energetic particles from 18:30 to 21:00 UT and from 20:40 to 23:10 UT on 1 January 2016, respectively, and found that the intensities of electrons with energies from tens of kiloelectron volts to $2 \mathrm{MeV}$ oscillated

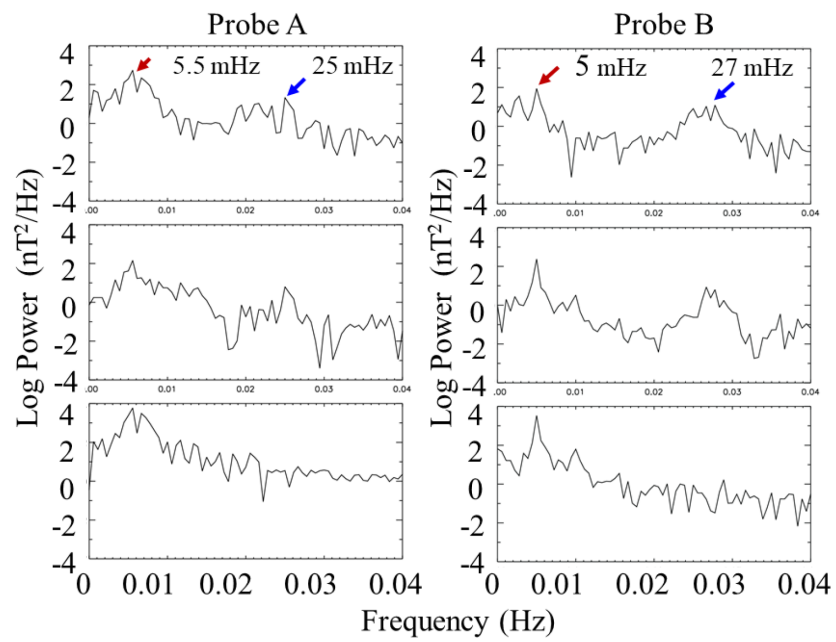

Figure 8. Fourier spectra calculated for the radial, azimuthal, and compressional components of the RBSP-A and RBSP-B magnetic field in 5 min sliding-average mean field-aligned coordinates from 19:30 to 20:00 UT and from 22:30 to 23:00 UT on 1 January 2016, respectively.

with Pc5 periods corresponding to those of the magnetic field. Figure 10a and b show an example of RBSP-A observations of electron fluxes (a) in the energy range of from 31.5 to $1704 \mathrm{keV}$ from 18:30 to 21:00 UT and (b) their expanded view for selected energies from 19:20 to 20:00 UT. The energetic electron fluxes oscillated out of phase with the compressional $\mathrm{Bz}$ component of Pc5 magnetic field pulsations and did not display any phase differences across all en- 
ergies. The depth of modulation (the peak to valley ratio) is greater for higher-energy electrons, consistent with the results by Liu et al. (2016), who interpreted similar observations in terms of mirror-mode waves. The lower-energy electron fluxes displayed more noticeable enhancements as a response to the compressions of the magnetosphere. Kivelson and Southwood (1985) noted that the maintenance of pressure balance in low-frequency compressional waves usually requires the presence of some pitch angle anisotropy, and the antiphase relation between the plasma and magnetic field pressures suggests that particle pitch angle distributions peak near $90^{\circ}$. Figure 11 presents RBSP-A and RBSP-B observations of pitch angle distributions for electrons with energies from 54 to $1060 \mathrm{keV}$ from 18:30 to 21:00 UT and from 20:40 to 23:10 UT on 1 January 2016, respectively. The figure confirms that pitch angle distributions peak near $90^{\circ}$. Furthermore, it shows that the electron intensities display quasiperiodic enhancements at all energies with the strongest at pitch angles near $90^{\circ}$.

\subsection{Double-frequency pulsations}

When RBSP-A and RBSP-B were in the vicinity of the geomagnetic equator, the compressional Pc5 pulsations displayed peculiar features indicating frequency doubling. The compressional components oscillated with a frequency twice that of the transverse component. Coleman (1970) was the first to report observations of such events in the geosynchronous magnetic field. Higuchi et al. (1986) called them harmonic structures when the first and second harmonics exhibited similar amplitudes and transitional structures when the amplitudes of the alternating peak were different. Takahashi (1987b) interpreted double-frequency oscillations in terms of a model invoking the second-harmonic structure of an antisymmetric standing wave in which the location of the equatorial node of field-aligned displacement oscillates in phase with the wave. Cheng and Qian (1994) presented a model for the magnetic field perturbations during the pulsations reported by Takahashi et al. (1987a, 1990). Figure 6 in the paper of Korotova et al. (2013) illustrates how lowlatitude spacecraft can observe two magnetic field strength enhancements per wave cycle when the equatorial node oscillates latitudinally up and down in phase with an antisymmetric compressional wave. Right at the equator the spacecraft observes identical amplitudes for the two compressions. At any other latitude the two compressions at the spacecraft will have different magnitudes and the imbalance between them increases when the spacecraft moves farther from the equator. Takahashi et al. (1987b) showed that a latitudinal shift of a fraction of a degree can turn a harmonic Bz structure into a nonharmonic structure. Spacecraft located far from the magnetic equator do not observe frequency doubling but just a single enhancement. Korotova et al. (2013) derived the latitudinal structure of the waves by invoking north-south sloshing of the low-latitude node.
Figure 12a and b present (a) RBSP-A and RBSP-B observations of double-frequency magnetic pulsations and (b) their locations in the X-Y GSM and X-Z SM planes. Dashed lines in Fig. 12a indicate intervals when the doublefrequency pulsations in $\mathrm{Bz}$ are most prominent: 20:45 to 20:54 UT at RBSP-A and 21:03 to 21:31 UT at RBSP-B in these line plots. However, the amplitudes of the second harmonic are generally much lower than those of the first harmonic. At these times, e.g., from 20:05 to 20:45 UT at RBSP-A and from 21:35 to 21:55 UT at RBSP-B, the secondharmonic compressions in $\mathrm{Bz}$ are barely perceptible in these line plots. Model predictions for the magnetic field perturbations associated with an equatorial node whose latitude oscillates in phase with an antisymmetric poloidal wave indicate that the ratio of the amplitudes of the first-to-second harmonic compressions should change with latitude, being $\sim 1$ at the average position of the low-latitude node and $\sim 0$ at and beyond the maximum latitude to which the oscillating node can reach (Takahashi et al., 1987b). To determine the meridional motion of the magnetic field node, we measured amplitudes of the first and second harmonics of the compressional pulsations. We found that RBSP-A observed ratios near 1 at $Z_{\mathrm{SM}}=\sim 0.08 \mathrm{Re}$, while RBSP-B observed ratios near 1 at $Z_{\mathrm{SM}}=\sim 0.10 \mathrm{Re}$. These are the locations where the southward-moving spacecraft pass through the mean positions of the equatorial node. Figure 12a shows that RBSP-A observed second harmonics from $Z_{\mathrm{SM}}=0.25$ to $0.04 \mathrm{Re}$, while RBSP-B observed them from $Z_{\mathrm{SM}}=0.19$ to $-0.08 \mathrm{Re}$. Consequently, we believe that the equatorial node oscillated with an amplitude of at least 0.15 to $0.18 \mathrm{Re}$. Note, however, that the ratio of the first-to-second harmonics does not show a smooth transition as the spacecraft move equatorward. Either the amplitude of the compressional pulsation or the meridional oscillation in the equatorial node varied in time, probably abruptly.

Figure 10a and $\mathrm{b}$ show that the compressional pulsations modulated energetic electrons observed by RBSP-A, and we should therefore expect to find the signatures of the doublefrequency pulsations not only in the magnetic field but also in the fluxes of particles. Takahashi et al. (1990) reported AMPTE/CCE (Charge Composition Explorer) observations of compressional Pc5 pulsations that exhibited harmonically related transverse and compressional magnetic oscillations that modulated the flux of medium-energy protons $(E>$ $10 \mathrm{keV}$ ) with double frequency but did not discuss the event in detail. We report the first evidence for meridional sloshing of the equatorial node in the simultaneous compressional Pc5 pulsations and variations of electron fluxes and electron densities observed by MagEIS and Hope, respectively. Figure 13 presents RBSP-A (left panel) and RBSP-B (right panel) electron fluxes for energies at 31.9 and $54.8 \mathrm{keV}$, electron densities, and the Bz component of the magnetic field in FAC from 19:00 to 21:00 UT and at RBSP-B from 20:46 to 22:10 UT. The panels in the bottom of Fig. 13 present expanded views of $20 \mathrm{~min}$ intervals with the double-frequency pulsations. 


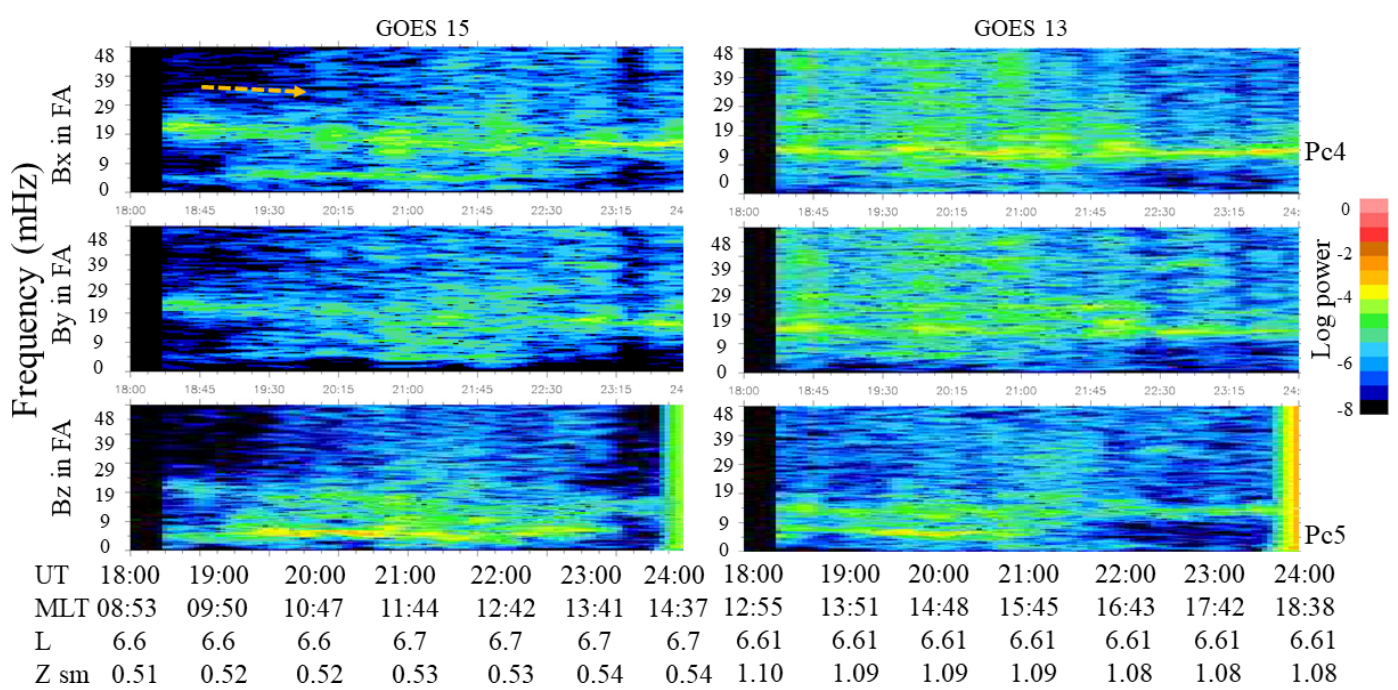

Figure 9. Three components of dynamic spectra of the magnetic field data at G-15 and G-13 from 18:00 to 24:00 UT on 1 January 2016. Beneath the panels are listed the universal time (UT), magnetic local time (MLT in SM), L, and Z (SM) in Earth radii.
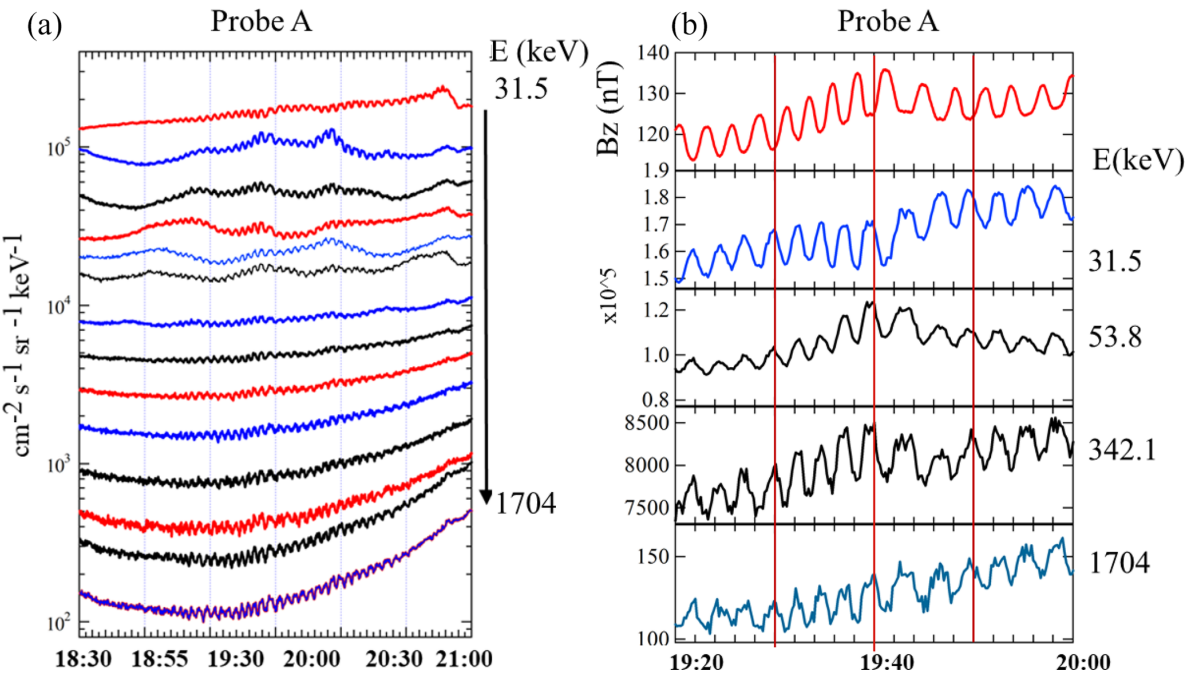

Figure 10. (a, b) RBSP-A observations of electron fluxes (a) in the energy range from 31.5 to $1704 \mathrm{keV}$ from 18:30 to 21:00 UT and (b) their expanded view for selected energies from 19:20 to 20:00 UT.

The Bz component of the magnetic field varies with double frequencies out of phase with the fluxes of electrons and densities. This study gives better insight into the nodal structure of the waves and helps to clarify their source.

\subsection{Testing Pc4-5 pulsation generation mechanisms}

We tested several causes for the Pc4-5 pulsations, including solar wind pressure pulses, the KH instability on the magnetopause, drift-bounce resonant particle interactions, and the mirror-mode instability. First, with the exception of the interval from 19:35 to 19:55 UT, the Wind observations shown in Fig. 1 provide no evidence for periodic solar wind drivers in the Pc5 range, be they density variations or IMF fluctua- tions, thus ruling out solar wind pressure pulses as the direct cause of the Pc4-5 pulsations. We then considered the possibility of $\mathrm{KH}$ waves. These waves are expected when the solar wind velocity is high and both the magnetosheath and magnetospheric magnetic fields lie transverse to the magnetosheath flow, i.e., on the flanks of the magnetosphere when the IMF points southward or in particular northward (e.g., Guo et al., 2010). As shown in Fig. 1, the solar wind velocity during the interval when the Pc5 events occurred was only moderate, $400-460 \mathrm{~km} / \mathrm{s}$. Furthermore, the IMF did not point either strongly northward or southward. Therefore, we conclude that the compressional Pc5 pulsations were excited by processes internal to the magnetosphere. 

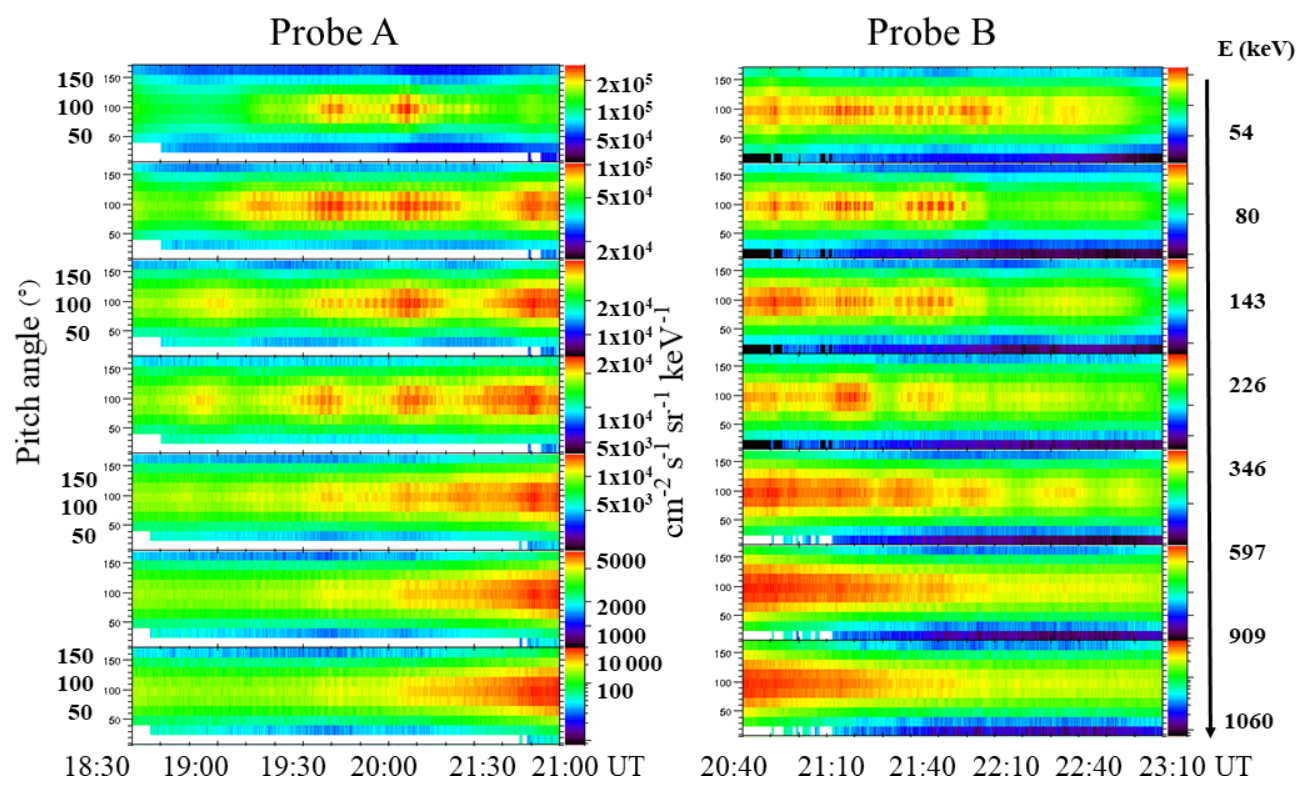

Figure 11. RBSP-A and RBSP-B observations of pitch angle distributions for electrons in the energy range from 54 to $1060 \mathrm{keV}$, from 18:30 to 21:00 UT and from 20:40 to 23:10 UT on 1 January 2016, respectively.

(a)

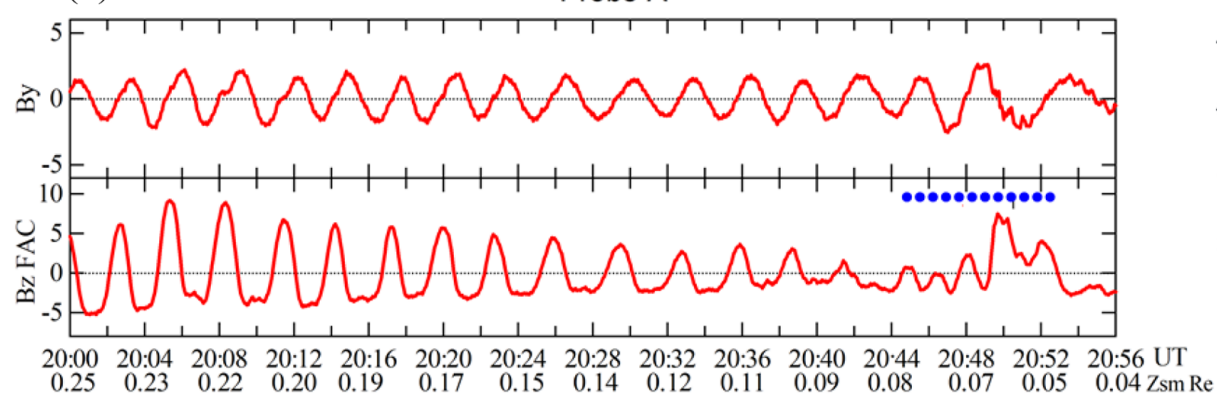

(b)

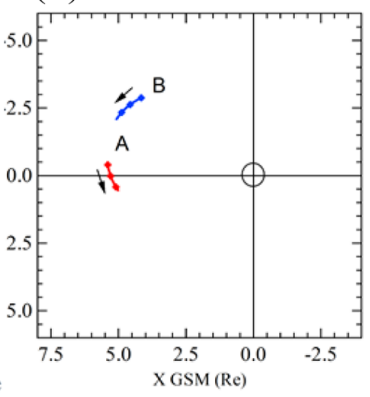

Probe B
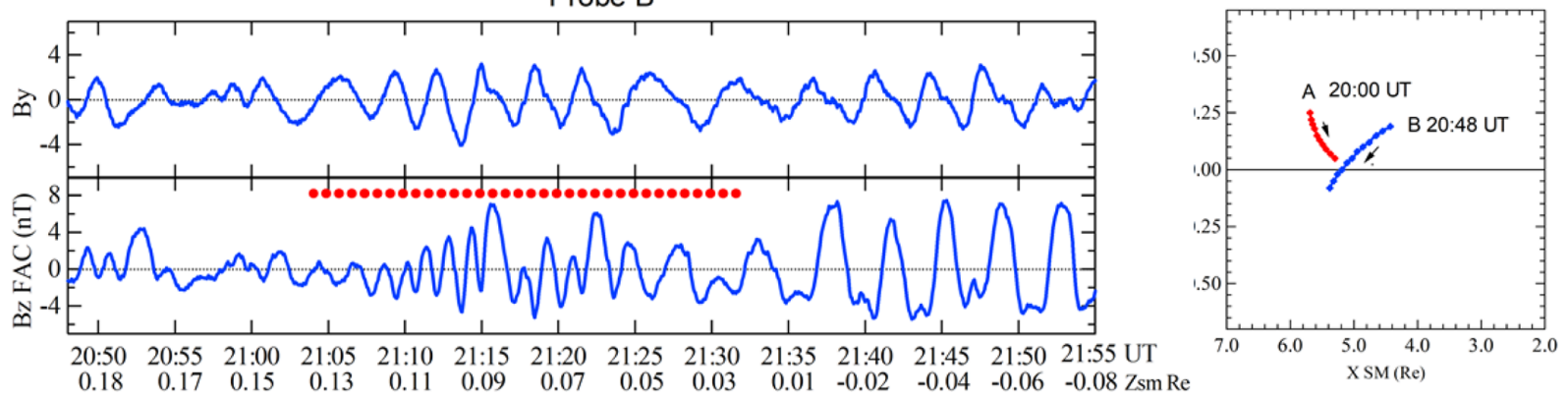

Figure 12. (a, b) RBSP-A and RBSP-B observations of double-frequency pulsations (a) from 20:00 to 20:56 UT and from 20:48 to 21:55 UT, respectively, and (b) their locations in the X-Y GSM and X-Z SM planes. Red and blue dashed lines mark the intervals with harmonic structure of double-frequency pulsations.

Southwood (1981) and Kivelson and Southwood (1985) described how the resonant drift-bounce interaction of particles with an azimuthally propagating wave generates largeamplitude ULF waves in an inhomogeneous background field. For this to happen, the wave frequency $\omega$ must satisfy the resonance condition:

$\omega-m \omega_{\mathrm{d}}-N \omega_{\mathrm{b}}=0$,

where $\omega_{\mathrm{d}}$ and $\omega_{\mathrm{b}}$ are the angular drift and bounce frequencies, respectively; $N$ is an integer; and $m$ is the azimuthal wave number. Southwood (1973) predicted that particle flux 
(a)

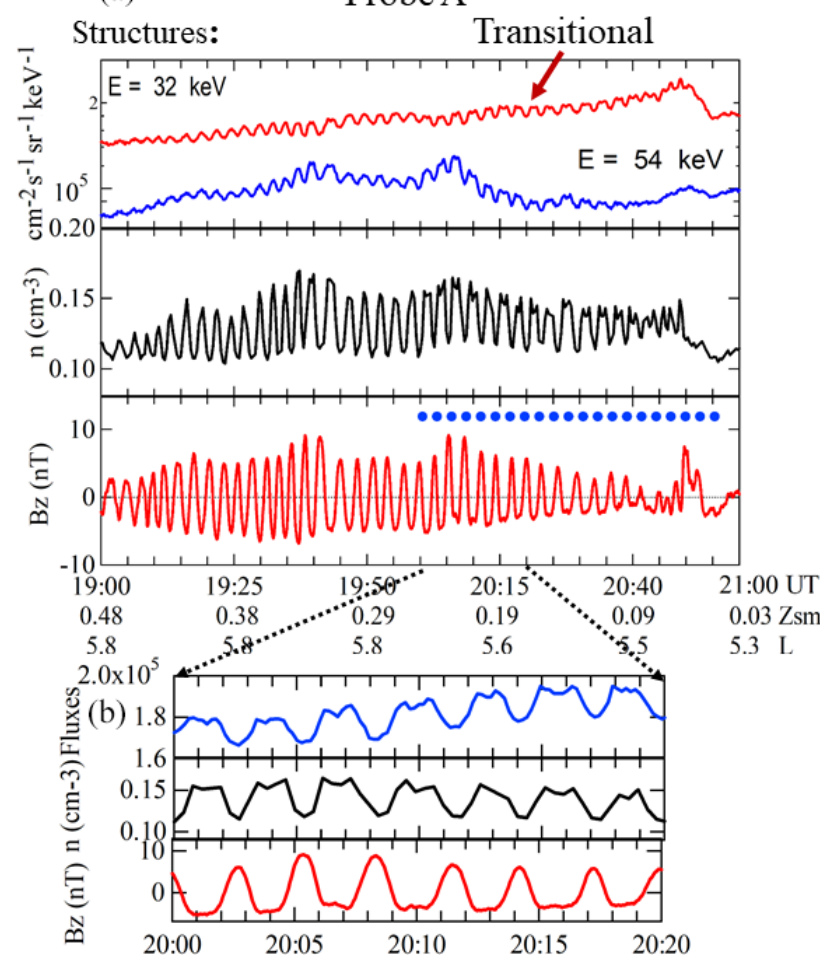

(c)

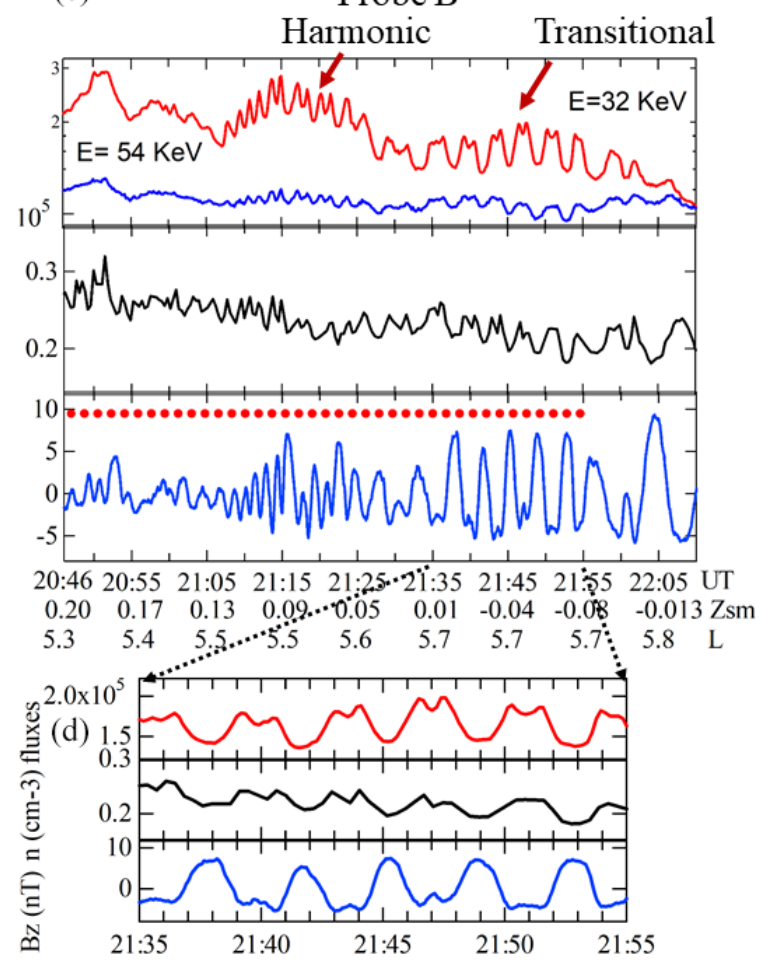

Figure 13. Panels for RBSP-A (a, b) and RBSP-B (c, d) present electron fluxes for energies at 31.9 and $54.8 \mathrm{keV}$ from EMFISIS, electron densities from HOPE and the Bz component of the magnetic field in field-aligned coordinates from MagEIS from 19:00 to 21:00 UT and from 20:46 to 22:10 UT, respectively. Dotted lines mark the intervals of observations of double-frequency pulsations. Panels (b, d) present expanded views of $20 \mathrm{~min}$ intervals with the double-frequency pulsations to better visualize their features.

oscillations just above and below the resonant energy should be $180^{\circ}$ out of phase. As Fig. 10a and b demonstrate, RBSPA did not observe any such phase reversal in the electrons as a function of energy. We exclude the drift-bounce resonance as the cause of these compressional Pc5 pulsations.

Finally, we examined the mirror instability criterion. The mirror instability is a kinetic phenomenon that occurs spontaneously in anisotropic high- $\beta$ plasmas when the ratio of perpendicular to parallel pressures is large (Southwood and Kivelson, 1993). The test for the mirror instability is approximately given as follows:

$\Gamma=1+\beta_{\perp}\left(1-T_{\perp} / T_{/ /}\right)<0$,

where $T_{/ /}$and $T_{\perp}$ are the plasma temperatures parallel and perpendicular, respectively, to the ambient magnetic field, and $\beta_{\perp}$ is the ratio of the perpendicular component of the thermal plasma pressure to the magnetic pressure. For our calculations, we obtained the magnetic field data from EMFISIS and thermal plasma pressures perpendicular and parallel to the magnetic field from RBSPICE. We used the density and temperature from HOPE to calculate the parallel and perpendicular thermal pressures within the energy range covered by this instrument, but we found these pressures to be small compared to those from RBSPICE. Consequently, our calcu- lations neglect the contributions from HOPE to the thermal pressures.

Figure 14a and $b$ show RBSP-A and RBSP-B plasma and magnetic field parameters characterizing the pulsations. The upper panels indicate that magnetic field and plasma pressures vary in antiphase during the Pc5 pulsations. However, the total pressure is not balanced as might be expected for mirror-mode waves. We suppose that this is because the RBSPICE (or even the RBSPICE + HOPE) plasma instruments do not observe the entire plasma distribution. Assuming that the total plasma pressure is proportional to the fraction that RBSPICE does observe, we scaled the thermal plasma pressures observed by RBSPICE upward to values that cause the sum of the magnetic and perpendicular thermal plasma pressure variations associated with the waves to be approximately constant during the intervals from 19:03 to 19:14 UT for RBSP-A and from 22:32 to 22:56 UT for RBSP-B. The upward scaling factors were 1.97 and 1.69 , respectively. We then applied these factors to both the perpendicular and parallel pressures. The third subpanels of Fig. 14a and b show the values of $\beta_{\perp}$ calculated from these scaled pressures. Shaded grey areas in the fourth subpanels show when the drift-mirror instability is satisfied $(<0)$. As the test for the mirror instability is satisfied throughout most of the intervals 
(a)

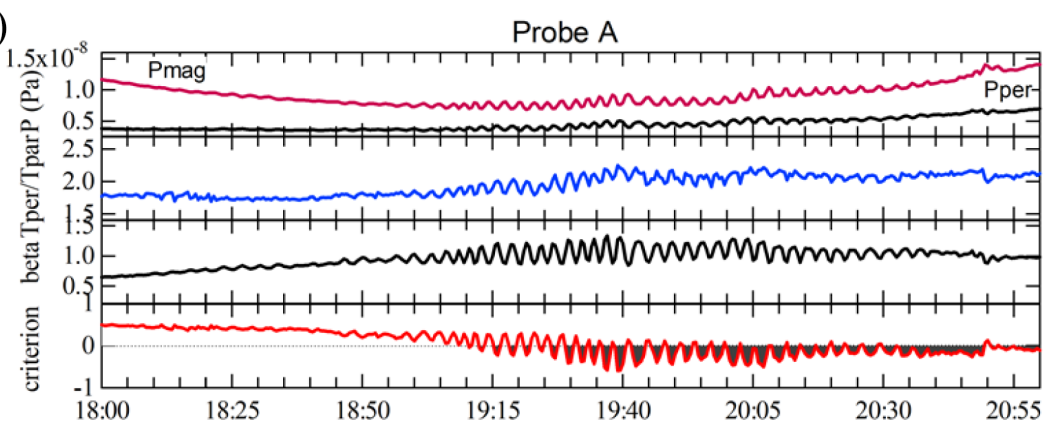

(b)

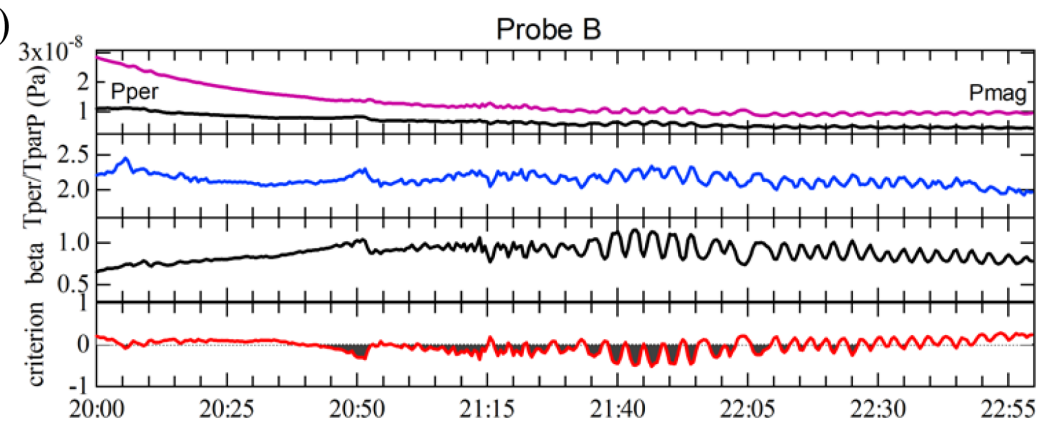

Figure 14. (a, b) RBSP-A and RBSP-B plasma and magnetic field parameters characterizing the pulsations. Subpanels in each panel show the magnetic pressure, perpendicular plasma pressure, the ratio of the plasma temperatures perpendicular and parallel to the magnetic field, beta, and the results for the mirror instability criterion on 1 January 2016. Shaded grey areas indicate the times when the drift-mirror instability is satisfied $(<1)$.

of enhanced temperature (pressure) anisotropy and $\beta>1$ at RBSP-A and RBSP-B, we attribute the compressional Pc5 pulsations observed on 1 January 2016 to the mirror instability.

\section{Conclusions}

We used Van Allen Probes and GOES multipoint magnetic field, electric field, plasma, and energetic particle observations to study the nature of compressional Pc5 pulsations at the end of a strong magnetic storm on 1 January 2016. From $\sim$ 19:00 to 23:02 UT the magnetosphere was compressed and transient increases of the total magnetic field strength occurred every 20-40 min. During this interval the spacecraft observed compressional Pc5 pulsations over a large longitudinal extent. The solar wind pressure enhancements initiated and/or amplified compressional wave activity in the dayside magnetosphere. The pulsations occupied the dayside magnetosphere from 5.26 to $6.6 \mathrm{Re}$ and from 09:56 to 15:20 MLT. Successive solar wind pressure increases and magnetospheric compressions enhanced the amplitude of Pc5 wave activity to values from 10 to $16 \mathrm{nT}$. The strongest amplitudes occurred prior to local noon. They were observed when the IMF cone angle was less than $45^{\circ}$. We studied the wave mode of the Pc5 pulsations and found that they had an antisymmetric structure.
The greatest spectral power densities observed at RBSPA and RBSP-B occurred in the north or south (Bz) component of the magnetic field at frequencies of $\sim 4.5-6.0 \mathrm{mHz}$. The two spacecraft observed similar frequencies, indicating that conditions within the dayside magnetosphere remained steady for a long time and over a broad region. Enhanced spectral power densities at frequencies of $\sim 22-29 \mathrm{mHz}$ in the radial $\mathrm{Bx}$ component can be attributed to the simultaneous generation of poloidal Pc4 pulsations by a different mechanism. The frequencies of the Pc4 pulsations diminished with increasing radial distance. The dominant frequencies for the compressional Pc5 pulsations observed by GOES resembled those observed by RBSP-A and RBSP-B, and we suppose that they were generated by the same sources. Pc4 pulsations observed by the GOES spacecraft displayed frequencies that were lower than those observed by RBSP-A and RBSP-B, since the GOES spacecraft were located further radially outward from Earth. Since the frequencies of the Pc4 pulsations depended on local time and radial distance from Earth, their sources must be more localized than those for the Pc5 pulsations.

When the spacecraft were in the vicinity of the geomagnetic equator, RBSP-A observed meridional sloshing of the equatorial wave node from $Z_{\mathrm{SM}}=0.25$ to $0.04 \mathrm{Re}$, while RBSP-B observed them from $Z_{\mathrm{SM}}=0.19$ to $-0.08 \mathrm{Re}$. Consequently, we believe that the motion of the meridional oscillation of the position of the equatorial node was at least 0.15 to $0.18 \mathrm{Re}$. We found that RBSP-A observed ratios near 
1 at $Z_{\mathrm{SM}}=\sim 0.08 \mathrm{Re}$, while RBSP-B observed ratios near 1 at $Z_{\mathrm{SM}}=\sim 0.10 \mathrm{Re}$. These were the locations where the southward-moving spacecraft RBSP-A and RBSP-B passed through the mean positions of the equatorial node at $Z_{\mathrm{SM}}=\sim$ $0.08 \mathrm{Re}$ and at $Z_{\mathrm{SM}}=\sim 0.10 \mathrm{Re}$, respectively. We report the first evidence for meridional sloshing of the equatorial node in the double-frequency variations of electron fluxes and electron density observed by MagEIS and HOPE, respectively.

The energetic particles observed by RBSP-A and RBSP$B$ showed a regular periodicity over a broad range of energies from tens of electron volts to $2 \mathrm{MeV}$ with periods corresponding to those of the compressional component of the ULF magnetic field. The electron intensities exhibited quasiperiodic enhancements at all energies with the most intense at pitch angles near $90^{\circ}$. The energetic electron fluxes oscillated out of phase with the magnetic field and did not display any phase shift across all energies. The depth of modulation was larger for higher-energy electrons. We searched for possible solar wind triggers and discussed generation mechanisms for the compressional Pc5 pulsations in terms of driftmirror instability and drift-bounce resonance. We interpret the compressional Pc5 waves in terms of drift-mirror instability.

Data availability. Data used in the paper are available publicly at https://cdaweb.gsfc.nasa.gov/istp_public/, last access: 1 March 2020. GOES data were obtained from http://satdat.ngdc.noaa.gov/ sem/goes/data/new_full/, last access: 2 January 2019. The electric field data were obtained from http://www.space.umn.edu/ rbspefw-data/ (Wygant and Breneman, 2017), last access: 30 January, 2020.

Author contributions. GK drafted and wrote the paper with participation of all coauthors. DS conceived the ideas. ME, ST, HS, and $\mathrm{CK}$ were consulted regarding the data analysis. RR contributed to software development. MB was consulted regarding the drift-mirror instability test.

Competing interests. The authors declare that they have no conflict of interest.

Acknowledgements. We thank the Van Allen Probes project and experiment teams for their efforts to provide high-quality observations. We acknowledge NASA GSFC's CDAWeb site for providing access to the observations.

Financial support. Galina Korotova was supported by NASA contract no. 80NSSC19K0440. Michael Balikhin is grateful to the UK Science and Technology Facilities Council (STFC) (grant ST/R000697/1). David Sibeck was supported by GSFC WBS
605745.04.01, corresponding to the Van Allen Probes project. The work by the EFW team at the University of Minnesota was supported by APL contract to UMN 922613 under a NASA contract to APL NAS5-01072.

Review statement. This paper was edited by Georgios Balasis and reviewed by two anonymous referees.

\section{References}

Blake, J. B., Carranza, P. A., Claudepierre, S. G., Clemmons, J. H., Crain Jr., W. R., Dotan, Y., Fennell, J. F., Fuentes, F. H., Galvan, R. M., George, J. S., Henderson, M. G., Lalic, M., Lin, A. Y., Looper, M. D., Mabry, D. J., Mazur, J. E., McCarthy, B., Nguyen, C. Q., O’Brien, T. P., Perez, M. A., Redding, M. T., Roeder, J. L., Salvaggio, D. J., Sorensen, G. A., Spence, H. E., Yi, S., and Zakrzewski, M. P.: The Magnetic Electron Ion Spectrometer (MagEIS) Instruments Aboard the Radiation Belt Storm Probes (RBSP) Spacecraft, Space Sci. Rev., 179, 383-421, https://doi.org/10.1007/s11214-013-9991-8, 2013.

Chen, L. and Hasegawa, A.: Kinetic theory of geomagnetic pulsations.1. Internal excitations by energetic particles, J. Geophys. Res., 96, 1503-1512, https://doi.org/10.1029/90JA02346, 1991.

Cheng, C. Z. and Lin, C. S.: Eigenmode analysis of compressional waves in the magnetosphere, Geophys. Res. Lett., 8, 884-887, https://doi.org/10.1029/GL014i008p00884, 1987.

Cheng, C. Z. and Qian, O.: Theory of ballooning-mirror mode instabilities for anisotropic pressure plasmas in the magnetosphere, J. Geophys. Res., 99, 11193-11210, https://doi.org/10.1029/94JA00657, 1994.

Coleman Jr., P. J.: Geomagnetic storms at ATS 1, in: Intercorrelated Satellite observations related to solar events, edited by: Manno, V. and Page, D. E., Intercorrelated Satellite Observations Related to Solar Events, Astrophysics and Space Science Library, Vol 19, Springer, Dordrecht, https://doi.org/10.1007/97894-010-3278-0_18, 1970.

Constantinescu, O. D., Glassmeier, K.-H., Plaschke, F., Auster, U., Angelopoulos, V., Baumjohann, W., Fornaçon, K.-H., Georgescu, E., Larson. D., Magnes, W., McFadden, J. P., Nakamura, R., and Narita, Y.: THEMIS observations of dusk side compressional Pc5 pulsations, J. Geophys. Res., 114, A00C25, https://doi.org/10.1029/2008JA013519, 2009.

Cummings, W. D., O’Sullivan, R. J., and Coleman Jr., P. J.: Standing Alfvén waves in the magnetosphere, J. Geophys. Res., 74, 778793, https://doi.org/10.1029/JA074i003p00778, 1969.

Dai, L., Takahashi, K., Wygant, J. R., Chen, L., Bonnell, J., Cattell, C. A., Thaller, S., Kletzing, C., Smith, C. W., MacDowall, R. J., Baker, D. N., Blake, J. B., Fennell, J., Claudepierre, S., Funsten, H. O., Reeves, G. D., and Spence, H. E.: Excitation of poloidal standing Alfven waves through drift resonance wave-particle interaction, Geophys. Res. Lett., 40, 4127-4132, https://doi.org/10.1002/grl.50800, 2013.

Elkington, S. R., Hudson, M .K., and Chan, A. A.: Resonant acceleration and diffusion of outer zone electrons in an asymmetric geomagnetic field, J. Geophys. Res., 108, 1116, https://doi.org/10.1029/2001JA009202, 2003. 
Engebretson, M., Glassmeier, K.-H., Stellmacher, M., Hughes, W. J., amd Lühr, H.: The dependence of high-latitude PcS wave power on solar wind velocity and on the phase of highspeed solar wind streams, J. Geophys. Res., 103, 26271-26283, https://doi.org/10.1029/97JA03143, 1998.

Guo, X. C., Wang, C., and Hu, Y. Q.: Global MHD simulation of the Kelvin-Helmholtz instability at the magnetopause for northward interplanetary magnetic field, J. Geophys. Res., 115, A10218, https://doi.org/10.1029/2009JA015193, 2010.

Hedgecock, P. C.: Giant Pc5 pulsations in the outer magnetosphere: A study of HEOS-1 data, Planet. Space Sci., 24, 921935, https://doi.org/10.1016/0032-0633(76)90003-9, 1976.

Higbie, P. R., Baker, D. N., Zwickl, R. D., Bellian, R. D., Asbridge, J. R., Fennell, J. F., Wilken, B., and Arthur, C. W.: The Global Pc5 Event of November 14-15, 1979, J. Geophys. Res., 87, 2337-2345, https://doi.org/10.1029/JA087iA04p02337, 1982.

Higuchi, T., Kokubun, S., and Ohtani, S.: Harmonic structure of compressional Pc5 pulsations at synchronous orbit, Geophys. Res. Lett., 13, 1101, https://doi.org/10.1029/GL013i011p01101, 1986.

Kepko, L. and Spence, H. E.: Observations of discrete, global magnetospheric oscillations directly driven by solar wind density variations, J. Geophys. Res., 108, 1257, https://doi.org/10.1029/2002JA009676, 2003.

Kivelson, M. G. and Southwood, D. J.: Charged particle behavior in low-frequency geomagnetic pulsations, 4. Compressional waves, J. Geophys. Res., 90, 1486-1498, https://doi.org/10.1029/JA090iA02p01486, 1985.

Kletzing, C. A., Kurth, W. S., Acuna, M., MacDowall, R. J., Torbert, R. B., Averkamp, T., Bodet, D., Bounds, S. R., Chutter, M., Connerney, J., Crawford, D., Dolan, J. S., Dvorsky R., Hospodarsky, G. B., Howard, J., Jordanova, V., Johnson, R. A., Kirchner, D. L., Mokrzycki, B., Needell, G., Odom, J., Mark, D., Pfaff Jr., Phillips, J. R., Piker, C. W., Remington, S. L., Rowland, D., Santolik, O., Schnurr, R., Sheppard, D., Smith, C. W., Thorne, R. M., and Tyler, J.: The Electric and Magnetic Field Instrument Suite and Integrated Science (EMFISIS) on RBSP, Space Sci. Rev., 179, 127-181, https://doi.org/10.1007/s11214-013-9993-6, 2013.

Korotova, G. I., Sibeck, D. G., Angelopoulos, V., and Walsh, W.: Themis observations of compressional poloidal pulsations in the dawnside magnetosphere: a case study, J. Geophys. Res., 118, 7665-7673, https://doi.org/10.1002/2013JA019360, 2013.

Kokubun, S.: Statistical characteristics of Pc5 waves at geostationary orbit, J. Geomag. Geoelectr., 37, 759-779, https://doi.org/10.5636/jgg.37.759, 1985.

Kremser, G., Korth, A., Feier, J. A., Wilken, B., Gurevich, A. V., and Amata, E.: Observations of quasi-periodic flux variations of energetic ions and electron associated with Pc5 geomagnetic pulsations, J. Geophys. Res., 86, 3345-3356, https://doi.org/10.1029/JA086iA05p03345, 1981.

Lanzerotti, L. J., Hasegawa, A., and Maclennan, C. G.: Drift mirror instability in the magnetosphere: Particle and field oscillations and electron heating, J. Geophys. Res., 74, 5565-5578, https://doi.org/10.1029/JA074i024p05565, 1969.

Lin, C. S., Parks, G. K., and Winckler J. R.: The 2to12-min quasi-periodic variation of 50-to 1000-keV trapped electron fluxes, J. Geophys. Res., 81, 4517-4523, https://doi.org/10.1029/JA081i025p04517, 1976.
Lin, C. S. and Parks, G. K.: The coupling of Alfven and compressional waves, J. Geophys. Res., 83, 2628-2636, https://doi.org/10.1029/JA083iA06p02628, 1978.

Liu, H., Zong, Q.-G., Zhou, X. Z., Fu, S. Y., Rankin, R., Wang, L.-H., Yuan C. J., Wang, Y. F., Baker, D. N., Blake, J. B., and Kletzing, C. A.: Compressional ULF wave modulation of energetic particles in the inner magnetosphere, J. Geophys. Res., 121, 6262-6276, https://doi.org/10.1002/2016JA022706, 2016.

Lin, R. P., Anderson, K. A., Ashford, S., Carlson, C., Curtis, D., Ergun, R., Larson, D., McFadden, J., McCarthy, M., Parks, G. K., Rème, H., Bosqued, J. M., Coutelier, J., Cotin, F., Wenzel, K.-P., Sanderson, T. R., Henrion, J., Ronnet, J. C., and Paschmann, G.: A three-dimensional (3-D) plasma and energetic particle experiment for the Wind spacecraft of the ISTP/GGS mission, Space Sci. Rev., 71, 125-153, https://doi.org/10.1007/BF00751328, 1995.

Lepping, R.P., Acuna, M.H., Burlaga, L.F., Farrell, W.M., Slavin, J. A., Schatfen, K. H., Mariani, E., Ness, N. E., Neubauer, E. M., Whang, Y. C., Byrnes J. B., Kennon R. S., Panetta, P. V., Scheifele J., and Worley, E. M.: The WIND Magnetic field investigation, Space Sci. Rev., 71, 207-229, https://doi.org/10.1007/BF00751330, 1995.

Mauk, B. H., Fox, N. J., Kanekal, S. G., Kessel, R. L., Sibeck, D. G., and Ukhorskiy, A.: Science objectives and rationale for the radiation belt storm probes mission, Space Sci. Rev., 179, 3-27, https://doi.org/10.1007/s11214-012-9908-y, 2012.

Mitchell, D. G., Lanzerotti, L. J., Kim, C. K., Stokes, M., Ho, G., Cooper, S., Ukhorskiy, A., Manweiler, J. W., Jaskulek, J., Haggerty, D. K., Brandt, P., Sitnov, M., Keika, K., Hayes, J. R., Brown, L. E., Gurnee, R. S., Hutcheson, J. C., Nelson, K. S., Paschalidis, N., Rossano, E., and Kerem, S.: Radiation belt storm probes ion composition experiment (RBSPICE), Space Sci. Rev., 179, 263-308, https://doi.org/10.1007/s11214-0139965-x, 2013.

Motoba, T., Kikuchi, T., Okuzawa, T., and Yumoto, K.: Dynamical response of the magnetosphere-ionosphere system to a solar wind dynamic pressure oscillation, J. Geophys. Res., 108, 1206, https://doi.org/10.1029/2002JA009696, 2003.

Nagano, H. and Araki, T.: Long-duration Pc5 pulsations observed by geostationary satellites, Geophys. Res. Lett., 10, 908-911, https://doi.org/10.1029/GL010i009p00908, 1983.

Pokhotelov, O. A., Pilipenko, V. A., Nezlina, I. M., Woch, J., and Kremser, G.: Excitation of high $\beta$ plasma instabilities at the geostationary orbit: Theory and observations, Planet. Space Sci., 34, 695-712, https://doi.org/10.1016/0032-0633(86)90124-8, 1986.

Samson, J. C., Harrold, B. G., Ruohoniemi, J. M., Greenwald, R. A., and Walker, A. D. M.: Field line resonance associated with MHD waveguides in the magnetosphere, Geophys. Res. Lett., 19, 441-444, https://doi.org/10.1029/92GL00116, 1992.

Sarris, T. E., Liu, W., Li, X., Kabin, K., Talaat, E. R., Rankin, R., Angelopoulos, V., Bonnell, J., and Glassmeier, K.-H.: THEMIS observations of the spatial extent and pressure-pulse excitation of field line resonances, Geophys. Res. Lett., 37, L15104, https://doi.org/10.1029/2010GL044125, 2010.

Singer, H. J., Matheson, L., Grubb, R., Newman, A., and Bouwer, S. D.: Monitoring space weather with the GOES magnetometers, in: SPIE Conference Proceedings, edited by: Washwell, E. R., vol. 2812, GOES-8 and Beyond SPIE, Bellingham, WA, USA, 299-308, 1996. 
Shen, X.-C., Shi, Q., Wang, B., Zhang, H., Hudson, M. K., Nishimura, Y., Hartinger, M. D., Tian, A., Zong, Q. G., Rae, I. J., and Degeling, A. W.: Dayside magnetospheric and ionospheric responses to a foreshock transient on 25 June 2008: 1. FLR observed by satellite and ground-based magnetometers, J. Geophys. Res., 123, 6335-6346, https://doi.org/10.1029/2018JA025349, 2018.

Southwood, D. J., Dungey, J. W., and Etherington, R. J.: Bounce resonance interaction between pulsations and trapped particles, Planet. Space Sci., 17, 349-361, https://doi.org/10.1016/00320633(69)90068-3, 1969

Southwood, D. J.: The behaviour of ULF waves and particles in the magnetosphere, Planet. Space Sci., 21, 53-65, https://doi.org/10.1016/0032-0633(73)90019-6, 1973.

Southwood, D. J.: Low Frequency Pulsation Generation by Energetic Particles, Advances in Earth and Planetary Sciences, vol 11. Springer, Dordrecht, 75-88, https://doi.org/10.1007/978-94009-8426-4_5, 1981.

Southwood, D. J. and Kivelson, M. G.: Mirror instability: 1. Physical mechanism of linear instability, J. Geophys. Res., 98, 91819187, https://doi.org/10.1029/92JA02837, 1993.

Spence, H. E., Reeves, G. D., Baker, D. N., Blake J. B., Bolton, M., Bourdarie, S., Chan, S. G. Claudpierre, S. G., Clemmons, J. H., Cravens, J. P., Elkington, S. R., Fennell, J. F., Friedel, R. H. W., Funsten, H. O., Goldstein, J., Green, J. C., Guthrie, A., Henderson, M. G., Horne, R. B., Hudson, M. K., Jahn, J.M., Jordanova, V. K., Kanekal, S. G., Klatt, B. W., Larsen, B. A., Li, X., MacDonald, E. A., Mann, I. R., Niehof, J., O'Brien, T. P., Onsager, T. G., Salvaggio, D., Skoug, R. M., Smith, S. S., Suther, L. L., Thomsen, M. F., and Thorne R. M.: Science goals and overview of the Energetic Particle, Composition, and Thermal Plasma (ECT) Suite on NASA's Radiation Belt Storm Probes (RBSP) Mission, Space Sci. Rev., 179, 311-336, https://doi.org/10.1007/s11214013-0007-5, 2013.

Sugiura, M. and Wilson, C. R.: Oscillation of the geomagnetic field lines and associated magnetic perturbations at conjugate points, J. Geophys. Res., 69, 1211-1216, https://doi.org/10.1029/JZ069i007p01211, 1964.

Takahashi, K. and Ukhorskiy, A. Y.: Timing analysis of the relationship between solar wind parameters and geosynchronous Pc5 amplitude, J. Geophys. Res., 113, A12204, 1-13, https://doi.org/10.1029/2008JA013327, 2008.

Takahashi, K., McPherron, R. L., and Hughes, W. J.: Multispacecraft observations of the harmonic structure of Pc34 magnetic pulsations, J. Geophys. Res., 89, 6758-6774, https://doi.org/10.1029/JA089iA08p06758, 1984.

Takahashi, K., Higbie, P. R., and Baker, D. N.: Azimuthal propagation and frequency characteristic of compressional Pc5 waves observed at geostationary orbit, J. Geophys. Res., 90, 1473-1485, https://doi.org/10.1029/JA090iA02p01473, 1985.
Takahashi, K., Fennell, J. F., Amata, E., and Higbie, P. R.: Field-aligned structure of the storm time Pc5 wave of November 14-15, 1979, J. Geophys. Res., 92, 5857-5864, https://doi.org/10.1029/JA092iA06p05857, 1987a.

Takahashi, K., Zanetti, L. J., Potemra, T. A., and Acuña, M. H.: A model for the harmonic of compressional Pc5 waves, Geophys. Res. Let., 14, 363-366, https://doi.org/10.1029/GL014i004p00363, 1987b.

Takahashi, K., Cheng, C. Z., McEntire, R. W., and Kistler, L. M.: Observation and theory of Pc5 waves with harmonically related transverse and compressional components, J. Geophys Res., 95, 977-989, https://doi.org/10.1029/JA095iA02p00977, 1990.

Takahashi, K., Glassmeier, K.-H., Angelopoulos, V., Bonnell, J., Nishimura, Y., Singer, H. J., and Russell, C. T.: Multisatellite observations of a giant pulsation event, J. Geophys. Res., 116, A11223, https://doi.org/10.1029/2011JA016955, 2011.

Vaivads, A., Baumjohann, W., Haerendel, G., Nakamura, R., Kucharek, H., Klecker, B., Lessard, M.R., Kistler, L. M., Mukai, T., and Nishida, A: Compressional Pc5 type pulsations in the morning plasma sheet, Ann. Geophys., 19, 311-320, https://doi.org/10.1029/2001JA900042, 2001.

Walker, A. D. M., Greenwald, R. A., Korth, A., and Kremser, G.: STARE and GEOS 2 observations of a storm time Pc5 ULF pulsation, J. Geophys. Res., 87, 9135-9146, https://doi.org/10.1029/JA087iA11p09135, 1982.

Wang, B., Nishimura, Y., Hietala, H., Shen, X.-C., Shi, Q., Zhang, H., Lyons. L., Zou, Y., Angelopoulos, V., Ebihara, Y., and Weatherwax, A.: Dayside magnetospheric and ionospheric responses to a foreshock transient on 25 June 2008: 2. 2-D evolution based on dayside auroral imaging, J. Geophys. Res., 123, 6347-6359, https://doi.org/10.1029/2017JA024846, 2018.

Wygant, J. and Brenemean, A.: RBSP/EFW Home - University of Minnesota, available at: http://www.space.umn.edu/ rbspefw-data, last access: 30 January 2020.

Wygant, J. R., Bonnell, J. W., Goetz, K., Ergun, R. E., Mozer, F. S., Bale, S. D., Ludlam, M., Turin, P., Harvey, P. R., Hochmann, R., Harps, K., Dalton, G., McCauley, J., Rachelson, W., Gordon, D., Donakowski, B., Shultz, C., Smith, C., Diaz-Aguado, M., Fischer, J., Heavner, S., Berg, P., Malaspina, D. M., Bolton, M. K., Hudson, M., Strangeway, R. J., Baker, D. N., Li, X., Albert, J., Foster, J. C., Chaston, C. C., Mann, I., Donovan, E., Cully, C. M., Cattell, C. A., Krasnoselskikh, V., Kersten, K., Breneman, A., and Tao, J. B.: The electric field and waves instruments on the radiation belt storm probes mission, Space Sci. Rev., 179, 183-220, https://doi.org/10.1007/s11214-013-0013-7, 2013.

Zhang, X. Y., Zong, Q.-G., Wang, Y. F., Zhang, H., Xie, L., Fu, S. Y., Yuan, C. J., Yue, C., Yang, B., and Pu, Z. Y.: ULF waves excited by negative/positive solar wind dynamic pressure impulses at geosynchronous orbit, J. Geophys. Res., 115, A10221, https://doi.org/10.1029/2009JA015016, 2010.

Zhu, X. M. and Kivelson, M. G.: Compressional ULF waves in the outer magnetosphere: 1. Statistical study. J. Geophys. Res., 96, 19451-19467, https://doi.org/10.1029/91JA01860, 1991. 